\title{
Efeitos de dois procedimentos de ensino para formação de classes seqüenciais ${ }^{1}$
}

\author{
Grauben José Alves de Assis \\ Maria Elizângela Carvalho Sampaio \\ Universidade Federal do Pará
}

\begin{abstract}
Resumo
O presente estudo buscou verificar o efeito de dois procedimentos de ensino sobre a formação de classes seqüenciais. Seis adolescentes com retardo mental de leve a moderado foram expostos a duas ordens de treino distintas em dois grupos. No Grupo 1, após exposição ao treino de sobreposição com pares de estímulos adjacentes (overlapping two stimuli sequences) com duas sequiências de cinco figuras, os participantes foram expostos aos testes de ordenação em pares não-adjacentes e de substitutabilidade de estímulos. Em seguida, foram expostos ao treino de encadeamento de duas sequiências com as mesmas figuras e aos testes anteriores. No Grupo 2, os participantes foram expostos aos mesmos procedimentos de ensino, porém na ordem inversa. No Grupo1, nos testes de substitutabilidade, um participante (RLS) respondeu com $100 \%$ de acerto. No Grupo 2, nos testes de substitutabilidade, todos os participantes responderam também com 100\% de acerto. Conclui-se que houve emergência de classes seqüenciais e evidências de que o treino por encadeamento foi mais eficiente porque os participantes responderem prontamente em todos os testes, independentemente da ordem de apresentação dos treinos. A presença dos estímulos na tela ao longo das tentativas de treino com o procedimento de encadeamento, parece ter contribuído para esses resultados.
\end{abstract}

Palavras-chave: Classes seqüenciais; encadeamento; sobreposição; retardo mental.

\section{Abstract \\ Effects of two teaching procedures in the classes sequential formation}

This study confirms the effect of two training procedures on the formation of sequential classes. Six mentally retarded individuals were exposed to two training conditions, each with two of five stimulus sequences, followed by verification tests. In group 1, training trials involved the presentation stimulus pairs where the subject touched one, and then the other. After learning criteria was reached, an overlapping pair was introduced, until the students were exposed to four pairs. Subsequent to training with each stimulus set, the subjects were tested on nonadjacent pairs, then substitutability tests. In group 2, the number of stimuli in training trials was increased one by one, until the number of sets reached five, these were also followed by verification tests. All subjects met the training criterion. In group 1, the results for nonadjacent tests were positive for all subjects; one subject (RLS) showed $100 \%$ performance in substitutability test. In group 2, the results for nonadjacent tests and substitutability were positive for all subjects. During training, the sequential procedure was more effective because the participants responded quickly in all nonadjacent test pairs and in substitutability tests. Trials that reversed the order of conditions were necessary to verify the effects of these procedures on sequential class formation.

Keywords: Sequential classes; chaining; overlapping two stimuli; mental retardation.

Alguns autores (Green, Stromer \& Mackay, 1993) propuseram uma análise alternativa para a emergência de classes de estímulos resultantes de contingências de reforçamento que favorecem o estabelecimento de sequiências. Esta proposta busca esclarecer o porquê de evitar tanto a abordagem de cadeias comportamentais quanto a abordagem de controle de estímulos condicionais para a compreensão de desempenhos emergentes de sequiências não ensinadas. Tal pressuposto sugere que a investigação considere a ênfase nas relações entre estímulos dentro de uma dada sequiência por meio da análise de seqüências ensinadas separadamente e por meio de testes comportamentais apropriados que avaliam se tais relações possuem as propriedades de uma relação ordinal: irreflexividade, assimetria, transitividade e conectividade. A irreflexividade pressupõe que, em uma relação ordinal, um evento qualquer não pode seguir ele mesmo, isto é, não reflexiva. Por exemplo, a relação A1 $\rightarrow$ A1 não é verdadeira. A propriedade da assimetria pressupõe que uma relação ordinal deve ser unidirecional; neste caso se $\mathrm{A} 2 \rightarrow \mathrm{A} 3$, então $\mathrm{A} 3 \rightarrow \mathrm{A} 2$ não pode ser considerado correta. Uma relação ordinal é considerada transitiva se, por exemplo, A2 $\rightarrow$ A3 e $\mathrm{A} 3 \rightarrow \mathrm{A} 4$, então $\mathrm{A} 2 \rightarrow \mathrm{A} 4$; note-se que apenas pares de estímulos que não são adjacentes dentro de séries ensinadas podem ser a base para a inferência desta propriedade. E, finalmente, a propriedade de conectividade pressupõe que uma relação existe se é correlata para todos os pares de estímulos dentro de um dado campo. Ressalta-se que relações deste tipo são necessárias, mas sua identificação não é suficiente para o arranjo do que há dentro de uma sequiência. $\mathrm{Na}$ conectividade, se $\mathrm{A} 1 \rightarrow \mathrm{A} 2 \rightarrow \mathrm{A} 3$, então $\mathrm{A} 1 \rightarrow \mathrm{A} 2$, $\mathrm{A} 1 \rightarrow \mathrm{A} 3$ e $\mathrm{A} 2 \rightarrow \mathrm{A} 3$. Como se vê, esta propriedade somente é inferida se todos os pares são possíveis, pares não adjacentes dos quais a transitividade é inferida e pares adjacentes. Esta proposta é 
basicamente uma expansão do paradigma de Sidman (1986, 1994) sobre as relações entre estímulos em classe de estímulos equivalentes.

\section{Táticas para a análise de relações estímulo- estímulo dentro das sequências}

Duas táticas têm sido freqüentemente usadas para estudar relações ordinais entre estímulos dentro de sequiências. Cada uma delas envolve um paradigma de treino específico seguido por uma série de testes para desempenhos seqüenciais que não são diretamente treinados.

\section{a) Treino de Seqüências com 5 Estímulos}

Um tipo de treino muito usado envolve a produção de uma sequiência de cinco estímulos usando um procedimento de encadeamento padrão. Uma seqüência com dois estímulos $(\mathrm{A} 1 \rightarrow \mathrm{A} 2)$ é estabelecida primeiramente e, em seguida, os estímulos remanescentes são adicionados sucessivamente. Então, sondas avaliam a produção de todas as sequiências com dois estímulos que fazem parte das seqüências ensinadas diretamente. Usualmente são estabelecidos dez seqüências com dois estímulos, em que $\mathrm{A} 1 \rightarrow \mathrm{A} 2$ é a primeira a ser ensinada diretamente. São programadas dez sondas, quatro com pares de estímulos adjacentes e seis com pares de estímulos não adjacentes.

\section{b) Treino com pares de estímulos adjacentes (overlapping two stimulus sequences)}

A segunda tática descrita por Green, Stromer e Mackay (1993) para estabelecer seqüências de respostas é semelhante aos procedimentos usados em estudos de inferência transitiva (ver por exemplo, Stromer \& Mackay, 1990, 1992, 1993). Em resumo, o treino estabelece inúmeras seqüências com pares de estímulos adjacentes (overlapping two-stimulus sequences), ao invés de única seqüência envolvendo cinco estímulos. Nesse treino, ensinam-se quatro seqüências adjacentes $\mathrm{A} 1 \rightarrow \mathrm{A} 2, \mathrm{~A} 2 \rightarrow \mathrm{A} 3, \mathrm{~A} 3 \rightarrow \mathrm{A} 4$, $\mathrm{A} 4 \rightarrow \mathrm{A} 5$. Primeiro, as sequiências $\mathrm{A} 1 \rightarrow \mathrm{A} 2$ e A2 $\rightarrow$ A3 são ensinadas e depois misturadas. Então, a seqüência A3 $\rightarrow$ A4 é ensinada sozinha, e todos os três tipos de tentativas ensinadas são misturados. A seguir A4 $\rightarrow$ A5 é ensinada sozinha. Finalmente, todos os quatro tipos de tentativas são misturados randomicamente, e o treino com sequiências mistas provenientes da linha de base continua até que o critério estabelecido tenha sido alcançado.

\section{A formação de classes seqüienciais}

Alguns estudos têm investigado se o treino de duas ou mais seqüências independentes estabelece classes de estímulos cujos membros ocuparam a mesma posição ordinal (primeiro, segundo, terceiro) em diferentes sequiências. Este tipo de classe de estímulos é usualmente conhecido como uma "classe de seqüências" (Lazar, 1977; Lima \& Assis, 2003; Sigurdardottir, Green \& Saunders, 1990). Seu desenvolvimento é inferido a partir dos desempenhos em testes que avaliam se estímulos de uma mesma posição ordinal em diferentes sequiências são substituíveis uns pelos outros em sequiências não ensinadas ou são relacionados condicionalmente através de um procedimento de emparelhamento ao modelo (matching to sample-MTS).

\section{Treino de duas ou mais sequiências independentes}

No procedimento que examina o desenvolvimento de classes de seqüências duas ou mais seqüências podem ser ensinadas separadamente, de acordo com as técnicas descritas anteriormente. Em seguida, sondas com seqüências não ensinadas diretamente, mas organizadas a partir dos membros de cada uma das sequiências já ensinadas (por exemplo, $\mathrm{A} 1=>\mathrm{A} 3$, $\mathrm{B} 2=>\mathrm{B} 4)$, podem prover alguma evidência da propriedade de assimetria. Porém, ainda mais informativa seria a análise de tentativas em que estímulos de uma sequiência não ensinada diretamente fossem apresentados de modo aleatório em cada tentativa, permitindo uma avaliação da substitutabilidade de estímulos que ocupam a mesma posição em diferentes sequiências ensinadas, o que indicaria a emergência de uma nova classe de seqüência.

A emergência de classes seqüenciais ainda pode ser avaliada da seguinte forma: Após as sequiências terem sido ensinadas separadamente, os participantes são submetidos a testes MTS que avaliam se os estímulos que ocuparam a mesma posição ordinal em seqüências independentes tornam-se condicionalmente correlatos. A justificativa para este teste é que a substitutabilidade que aparece nas sondas com seqüências mistas indica que os estímulos de uma classe sequiencial também são membros de uma classe de equivalência. Resultados positivos foram obtidos com adultos normais (Sigurdardottir \& cols, 1990), um indivíduo com lesão cerebral (Stromer, Mackay, Cohen, \& Stoddard, 1993) e portadores de necessidades educacionais especiais (Sampaio \& Assis, no prelo), este último através de um treino por encadeamento de respostas. Entretanto, esses resultados não foram replicados quando os participantes foram expostos ao treino por encadeamento sob controle condicional (Portella, Sampaio, Baptista \& Assis, 2003).

Um estudo recente conduzido por Holcomb, Stromer e Mackay (1997) mostrou que crianças normais do pré-escolar foram capazes de responder seqüencialmente a três ou mais estímulos, após serem expostas ao treino de sobreposição com pares de estímulos adjacentes (overlapping two stimulus sequences) Os resultados mostraram também que relações de transitividade (responder seqüencialmente a pares de estímulos não adjacentes) foram obtidas, mesmo na ausência de qualquer mediação verbal. Os autores chamaram atenção principalmente para a ordem de treino em que as seqüências foram ensinadas.

Portanto, para explicitar os efeitos da ordem de treino, duas contingências de reforçamento foram 
programadas. A primeira com um procedimento de ensino de sobreposição com pares de estímulos adjacentes e a segunda através de um treino por encadeamento de respostas.

\section{Participantes}

\section{MÉTODO}

Seis alunos portadores de deficiência mental leve de uma unidade especializada em educação especial (SEDUC-PARÁ) foram recrutados a partir de contatos pessoais. Após entrevista com os pais ou responsáveis, quando assinavam um Termo de Compromisso concordando com a participação do aluno no estudo, o experimentador entrevistava o professor que orientava o aluno em sala. Todos foram informados de que se tratava de um estudo sobre método de ensino. No final de cada sessão experimental, os participantes recebiam um "brinde", independentemente do seu desempenho.

\section{Ambiente experimental e apparatus}

Um microcomputador (IBM PENTIUM de 133 $\mathrm{MHz}$ ), com tela sensível ao toque, controlava a apresentação dos estímulos, números de tentativas, número de posições de cada estímulo na tela e registro das respostas corretas e incorretas. O programa foi elaborado em linguagem VISUAL BASIC 5.0 por João Vicente Cunha Nascimento e desenvolvido especialmente para esta pesquisa. O estudo foi conduzido em uma sala da Psicologia de uma escola pública medindo aproximadamente $6 \mathrm{~m}^{2}$, climatizada e com relativo isolamento acústico. Cada sessão durava 30 minutos, no máximo.

\section{Estímulos}

Foram utilizados dois conjuntos de estímulos: aves (X) e mamíferos (W), conforme mostra a Figura 1. Cada estímulo, medindo aproximadamente $2 \mathrm{~cm} \mathrm{x}$ $2 \mathrm{~cm}$, era apresentado no interior de "janelas" que permaneciam disponíveis na tela do computador.
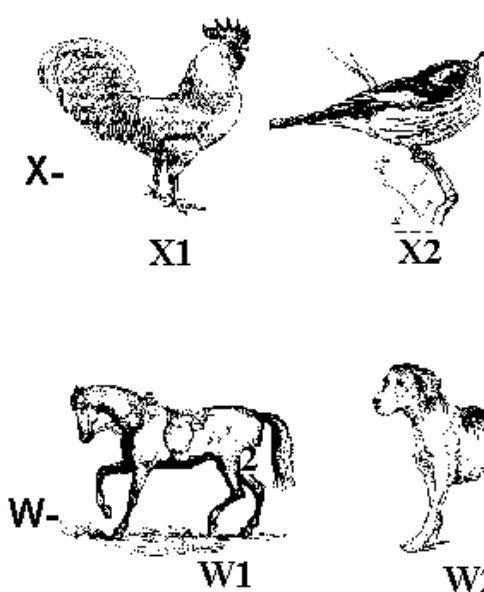

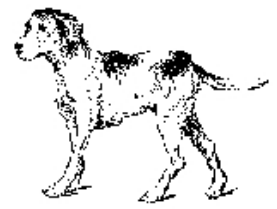

W2
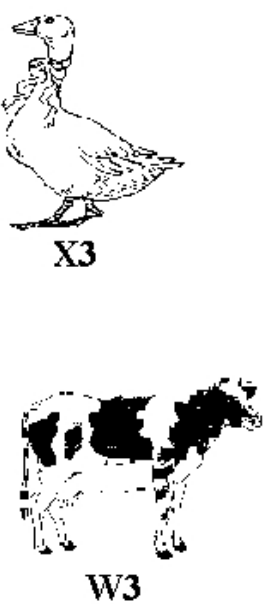
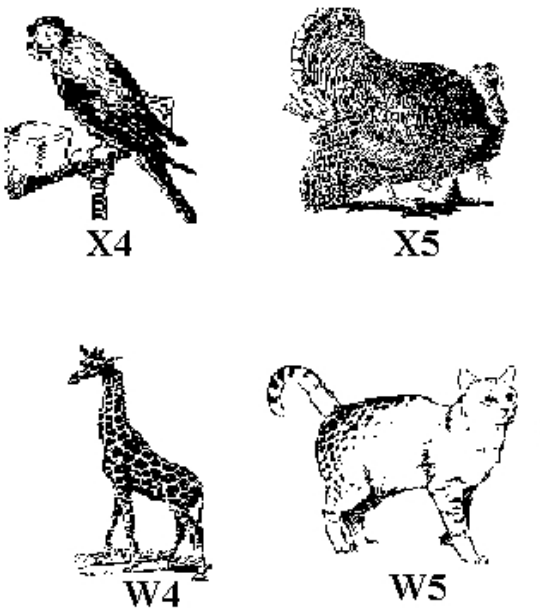

Figura 1 - Estímulos usados no estudo.

\section{PROCEDIMENTO}

Foi aplicado o Peabody Picture Vocabulary TestRevisited (Dunn \& Dunn, 1981) que teve por objetivo avaliar o repertório dos participantes quanto ao atraso no desenvolvimento fornecido pelo Manual Diagnóstico de Transtorno Mental (DSM-IV-edição revisada, 2000). A Tabela 1 apresenta cada um dos participantes por sexo, idades cronológicas e mentais respectivamente, no início do estudo.

Tabela 1 - Sexo, idade cronológica e mental de cada participante

\begin{tabular}{cccc}
\hline Participante & Sexo & Idade Cronológica & Idade Mental \\
\hline RUG & Masculino & 22 anos e 9 meses & $\begin{array}{c}5 \text { anos e 6 } \\
\text { meses }\end{array}$
\end{tabular}

$\begin{array}{cccc}\text { HSN } & \text { Masculino } & 18 \text { anos e 4 meses } & \begin{array}{c}4 \text { anos e } 6 \\ \text { meses } \\ 5 \text { anos e } 1 \\ \text { mês }\end{array} \\ \text { ELS } & \text { Feminino } & 17 \text { anos e 1 mês } & \begin{array}{c}5 \text { anos e 10 } \\ \text { meses }\end{array} \\ \text { CEL } & \text { Feminino } & 20 \text { anos e 5 meses } & \begin{array}{c}4 \text { anos e 11 } \\ \text { meses }\end{array} \\ \text { ETS } & \text { Masculino } & 14 \text { anos e 6 meses } & \begin{array}{c}3 \text { anos e 10 } \\ \text { meses }\end{array}\end{array}$

Dois grupos experimentais com 3 alunos cada foram distribuídos aleatoriamente. O Grupo 1 iniciou a tarefa com o procedimento de ensino de sobreposição com pares de estímulos adjacentes e em seguida os participantes foram expostos ao 
procedimento de treino por encadeamento. O Grupo 2 iniciou o treino com o procedimento de encadeamento e depois os participantes foram expostos ao treino de sobreposição com pares de estímulos adjacentes. Ou seja, cada participante foi exposto aos dois procedimentos de ensino, sob seu próprio controle (Sidman, 1960).

\section{Grupo 1}

Três fases estavam previstas: na Fase 1, o participante era ensinado a ordenar um conjunto de estímulos, a partir de um treino com pares de estímulos adjacentes. Após alcançar o critério de acerto (três vezes consecutivas sem erro) com os cinco estímulos em seqüência, os participantes eram expostos aos testes com os estímulos do conjunto " $\mathrm{X}$ ". Todos os estímulos eram apresentados na "área de escolha" (parte inferior do vídeo) da tela do computador. O participante tocava na parte da tela onde os estímulos eram apresentados até que nenhum mais restasse na "área de escolha". A tarefa do participante era tocar os estímulos, um após o outro. Toda vez que ele tocava na figura esta se deslocava da "área de escolha" para a "área de construção" (parte superior do vídeo) e as demais figuras eram reapresentadas na "área de escolha" em posição aleatória. Uma animação gráfica aparecia na tela juntamente com um som reproduzindo aplausos. Além disso, o experimentador dizia "Parabéns"!, "Muito bem!, você acertou"! ou "legal"!. Caso uma outra resposta fosse emitida, a tela se embranquecia por $2 \mathrm{~s}$ (intervalo entre tentativas) e as mesmas figuras eram reapresentadas na "área de escolha" aleatoriamente. Em seguida, um teste de seqüenciação era aplicado. Nesta condição, todos os estímulos do conjunto " $X$ " eram apresentados simultaneamente na área de escolha. Agora, o participante deveria responder tocando uma figura após a outra. Nessa tarefa de testes, em extinção, caso o participante não respondesse corretamente, na primeira exposição, era exposto mais uma vez ao teste.

No teste com para pares de estímulos não adjacentes, por exemplo, os estímulos do conjunto "X" eram apresentados na "área de escolha" aos pares,

Tabela 2 - Sumário dos procedimentos de treino e testes com overlapping two stimulus sequences, com tipo e número de tentativas, critério de acerto e probabilidade de reforços

\begin{tabular}{|c|c|c|c|c|c|}
\hline Fases & $\begin{array}{c}\text { Tipo de Tentativa } \\
\text { de Treino }\end{array}$ & Tipo de Tentativa de Testes & $\begin{array}{l}\text { Número de } \\
\text { Tentativas }\end{array}$ & $\begin{array}{l}\text { Critério de } \\
\text { Acerto }\end{array}$ & $\begin{array}{l}\text { Probabilidade de } \\
\text { Reforços }\end{array}$ \\
\hline \multirow{6}{*}{$\begin{array}{l}1 \text { - Pares de estímulos } \\
\text { do conjunto } X\end{array}$} & & & & $100 \%$ & $1 / 1$ \\
\hline & $(\mathrm{X} 1 \rightarrow \mathrm{X} 2)$ & & $3 / 3$ & & \\
\hline & $(X 2 \rightarrow X 3)$ & & $3 / 3$ & $100 \%$ & $1 / 1$ \\
\hline & $(X 3 \rightarrow X 4)$ & & $3 / 3$ & $100 \%$ & $1 / 1$ \\
\hline & $(X 4 \rightarrow X 5)$ & & $3 / 3$ & $100 \%$ & $1 / 1$ \\
\hline & & $\left(\mathrm{X}_{1} \rightarrow \mathrm{X}_{2} \rightarrow \mathrm{X}_{3} \rightarrow \mathrm{X}_{4} \rightarrow \mathrm{X}_{5}\right)$ & $1 / 2$ & - & - \\
\hline
\end{tabular}

em cada tentativa, por exemplo: $\mathrm{X} 1 \rightarrow \mathrm{X} 3$, ou $\mathrm{X} 1 \rightarrow \mathrm{X} 4$ e assim sucessivamente para cada um dos quatro pares de estímulos não adjacentes restantes, $\mathrm{X} 1 \rightarrow \mathrm{X} 5$, $\mathrm{X} 2 \rightarrow \mathrm{X} 4 ; \mathrm{X} 2 \rightarrow \mathrm{X} 5$ e X3 $\rightarrow \mathrm{X} 5$ (ver Tabela 2).

Na fase 2, o mesmo procedimento foi adotado com o conjunto de estímulos "W".

Na fase 3 um teste de substitutabilidade com os estímulos dos conjuntos "X" e "W" foi conduzido, por exemplo, $\quad \mathrm{X} 1 \rightarrow \mathrm{W} 2 \rightarrow \mathrm{X} 3 \rightarrow \mathrm{W} 4 \rightarrow \mathrm{X} 5 \quad$ ou ainda $\mathrm{W} 1 \rightarrow \mathrm{X} 2 \rightarrow \mathrm{W} 3 \rightarrow \mathrm{X} 4 \rightarrow \mathrm{W} 5$.

Em seguida, os participantes eram expostos ao treino com encadeamento de respostas, conforme pode ser observado na Tabela 3. Nesse treino, a topografia de resposta era a mesma do procedimento anterior, tocar levemente na tela do computador, em duas ou mais figuras em seqüência. Três fases foram programadas (ver Tabela 3). Os estímulos eram introduzidos gradativamente na "área de escolha". Por exemplo: X1 e X2, em seguida, X1, X2, X3. Depois, $\mathrm{X} 1, \mathrm{X} 2, \mathrm{X} 3$ e $\mathrm{X} 4$ ou ainda $\mathrm{X} 1, \mathrm{X} 2, \mathrm{X} 3, \mathrm{X} 4$ e X5. Após cada resposta correta (responder na seqüência programada, primeiro X1 e em seguida $\mathrm{X} 2$, por exemplo), uma animação gráfica e a reprodução de um som de aplausos eram apresentadas na tela, e a figura se deslocava para a "área de construção". Após todas as figuras terem sido apresentadas e o participante ter respondido à sequiência com todos os cinco estímulos (ver Figura 1), as figuras eram apresentadas simultaneamente na área de escolha e o participante deveria responder agora em extinção (teste de sequenciação). Em seguida, testes com pares de estímulos não adjacentes eram aplicados.

Na fase 2, o mesmo procedimento de treino e teste com pares não adjacentes foi adotado com o conjunto de estímulos "W". Na fase 3, testes de substitutabilidade com os dois conjuntos de estímulos também foram aplicados em extinção.

As Tabelas 2 e 3 apresentam todas as tentativas de treino e testes, número de tentativas, critério de acerto e probabilidade de reforços com os dois procedimentos de ensino. 
2 - Pares de estímulos do conjunto W

$\begin{array}{ll}X 1 \rightarrow X 3 & 1 / 2 \\ X 1 \rightarrow X 4 & 1 / 2 \\ X 1 \rightarrow X 5 & 1 / 2 \\ X 2 \rightarrow X 4 & 1 / 2 \\ X 2 \rightarrow X 5 & 1 / 2 \\ X 3 \rightarrow X 5 & 1 / 2\end{array}$

\begin{tabular}{|c|c|c|c|c|c|}
\hline & $(\mathrm{W} 1 \rightarrow \mathrm{W} 2)$ & & $3 / 3$ & $100 \%$ & $1 / 1$ \\
\hline & $(\mathrm{W} 2 \rightarrow \mathrm{W} 3)$ & & $3 / 3$ & $100 \%$ & $1 / 1$ \\
\hline & (W3 $\rightarrow$ W4) & & $3 / 3$ & $100 \%$ & $1 / 1$ \\
\hline & (W4 $\rightarrow$ W5) & & $3 / 3$ & $100 \%$ & $1 / 1$ \\
\hline & & $\underset{(\mathrm{W} 1}{ } \rightarrow \mathrm{W} 2 \underset{\mathrm{W} 5)}{\rightarrow} \mathrm{W}_{3} \rightarrow \mathrm{W} 4 \rightarrow$ & $1 / 2$ & - & - \\
\hline & & $\mathrm{W} 1 \rightarrow \mathrm{W} 3$ & $1 / 2$ & - & - \\
\hline & & $\mathrm{W} 1 \rightarrow \mathrm{W} 4$ & $1 / 2$ & & \\
\hline & & $\mathrm{W} 1 \rightarrow \mathrm{W} 5$ & $1 / 2$ & & \\
\hline & & $\mathrm{W} 2 \rightarrow \mathrm{W} 4$ & $1 / 2$ & & \\
\hline & & W2 $\rightarrow$ W5 & $1 / 2$ & & \\
\hline & & W3 $\rightarrow$ W5 & $1 / 2$ & & \\
\hline $\begin{array}{l}3 \quad-\quad \text { Teste } \\
\text { substitutabilidade } \\
\text { os conjuntos }\end{array}$ & $\begin{array}{r}\text { de } \\
\text { com } \\
\text { de }\end{array}$ & & & & \\
\hline estímulos $\rangle$ & & $\mathrm{X}_{1} \rightarrow \mathrm{W}_{2} \rightarrow \mathrm{X}_{3} \rightarrow \mathrm{W}_{4} \rightarrow \mathrm{X}_{5}$ & $1 / 2$ & - & - \\
\hline & & $\mathrm{W}_{1} \rightarrow \mathrm{X} 2 \rightarrow \mathrm{W}_{3} \rightarrow \mathrm{W}_{4} \rightarrow \mathrm{W} 5$ & $1 / 2$ & & \\
\hline
\end{tabular}

Tabela 3 - Sumário dos procedimentos de treino e testes com encadeamento, com tipo e número de tentativas, critério de acerto e probabilidade de reforços

\begin{tabular}{|c|c|c|c|c|}
\hline $\begin{array}{l}\text { Seqüências das Fases de } \\
\text { Treino e Testes }\end{array}$ & Tipo de tentativa & $\begin{array}{l}\text { Número de } \\
\text { Tentativas }\end{array}$ & $\begin{array}{l}\text { Critério de } \\
\text { Acerto }\end{array}$ & $\begin{array}{l}\text { Probabilidade } \\
\text { de Reforços }\end{array}$ \\
\hline \multirow{4}{*}{$\begin{array}{l}\text { Fase 1: Treino por encadeamento do } \\
\text { conjunto } X\end{array}$} & $\mathrm{X} 1 \rightarrow \mathrm{X} 2$ & $6 / 6$ & $100 \%$ & $1 / 1$ \\
\hline & $\mathrm{X}_{1} \rightarrow \mathrm{X}_{2} \rightarrow \mathrm{X}_{3}$ & $9 / 9$ & $100 \%$ & $1 / 1$ \\
\hline & $\mathrm{X}_{1} \rightarrow \mathrm{X}_{2} \rightarrow \mathrm{X}_{3} \rightarrow \mathrm{X}_{4}$ & $12 / 12$ & $100 \%$ & $1 / 1$ \\
\hline & $\mathrm{X} 1 \rightarrow \mathrm{X} 2 \rightarrow \mathrm{X} 3 \rightarrow \mathrm{X}_{4} \rightarrow \mathrm{X}_{5}$ & $15 / 15$ & $100 \%$ & $1 / 1$ \\
\hline \multirow{3}{*}{$\begin{array}{l}\text { Fase 1a: Teste de Sequenciação do } \\
\text { conjunto X }\end{array}$} & & & & \\
\hline & $\mathrm{X}_{1} \rightarrow \mathrm{X}_{2} \rightarrow \mathrm{X}_{3} \rightarrow \mathrm{X}_{4} \rightarrow \mathrm{X}_{5}$ & $1 / 2$ & - & - \\
\hline & $\mathrm{X} 1 \rightarrow \mathrm{X} 3$ & $1 / 2$ & - & - \\
\hline \multirow{6}{*}{$\begin{array}{l}\text { Fase 1b: Teste com pares de estímulos } \\
\text { não- adjacentes do conjunto X. }\end{array}$} & $\mathrm{X} 1 \rightarrow \mathrm{X} 4$ & $1 / 2$ & & \\
\hline & $\mathrm{X} 1 \rightarrow \mathrm{X} 5$ & $1 / 2$ & & \\
\hline & $X 2 \rightarrow X 4$ & $1 / 2$ & & \\
\hline & $X 2 \rightarrow X 5$ & $1 / 2$ & & \\
\hline & $X 3 \rightarrow X 5$ & $1 / 2$ & & \\
\hline & $W_{1} \rightarrow W 2$ & $6 / 6$ & $100 \%$ & $1 / 1$ \\
\hline \multirow{3}{*}{$\begin{array}{l}\text { Fase2: Treino por encadeamento com } \\
\text { estímulos do conjunto W }\end{array}$} & $W_{1} \rightarrow W_{2} \rightarrow W_{3}$ & $9 / 9$ & $100 \%$ & $1 / 1$ \\
\hline & $W_{1} \rightarrow W_{2} \rightarrow W_{3} \rightarrow W_{4}$ & $12 / 12$ & $100 \%$ & $1 / 1$ \\
\hline & $\mathrm{W}_{1} \rightarrow \mathrm{W}_{2} \rightarrow \mathrm{W}_{3} \rightarrow \mathrm{W}_{4} \rightarrow \mathrm{W}_{5}$ & $15 / 15$ & $100 \%$ & $1 / 1$ \\
\hline
\end{tabular}


Fase 2a: Teste de Sequenciação do conjunto W

$\mathrm{W} 1 \rightarrow \mathrm{W} 2 \rightarrow \mathrm{W}_{3} \rightarrow \mathrm{W}_{4} \rightarrow \mathrm{W} 5$

$\mathrm{W} 1 \rightarrow \mathrm{W} 3$

Fase 2b: Teste com pares de estímulos não-adjacente do conjunto W

$\mathrm{W} 1 \rightarrow \mathrm{W} 4$

W $1 \rightarrow$ W5
W2 $\rightarrow$ W4

W2 $\rightarrow$ W5

W3 $\rightarrow$ W5

Fase 3: Teste de substitutabilidade com os conjuntos de estímulos X e W
$\mathrm{X} 1 \rightarrow \mathrm{W} 2 \rightarrow \mathrm{X} 3 \rightarrow \mathrm{W} 4 \rightarrow \mathrm{X} 5$

$\mathrm{W}_{1} \rightarrow \mathrm{X} 2 \rightarrow \mathrm{W}_{3} \rightarrow \mathrm{W}_{4} \rightarrow \mathrm{W}_{5}$

\section{Grupo 2}

A mesma seqüência de treino e testes descrita para o Grupo 1 foi utilizada para este grupo, porém, os participantes iniciavam com o procedimento de encadeamento de respostas e em seguida eram expostos ao treino de sobreposição com pares de estímulos adjacentes.

\section{RESULTADOS E DISCUSSÃO}

Todos os participantes alcançaram o critério de acerto em ambos os grupos experimentais, ou seja, independentemente da ordem de treino. Nos testes com pares de estímulos não adjacentes, cinco participantes, dois do Grupo 1 (RUG e HSN) e os três

Tabela 4 - Número de respostas corretas pelo número máximo de tentativas durante o treino e testes com pares de estímulos adjacentes e não adjacentes e testes de substitutabilidade

GRUPO 1

SOBREPOSIÇÃO

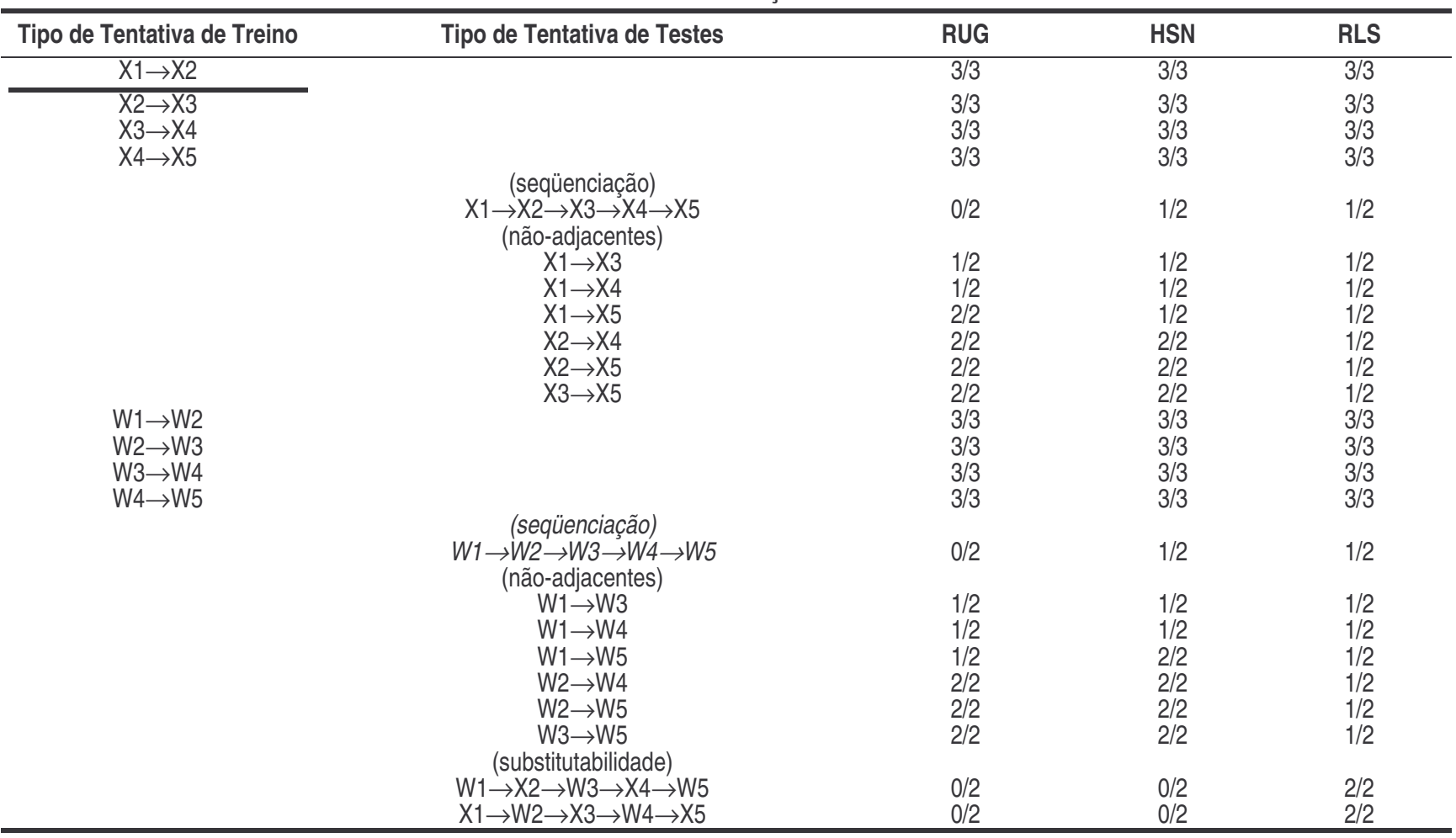

do Grupo 2 (EFS, CEL e ETS) precisaram de uma segunda exposição ao bloco de testes, ou seja, responderam corretamente com atraso (ver Tabelas 4 estímulos adjacentes. Somente um participante respondeu prontamente. $\mathrm{Na}$ fase de testes de substitutabilidade, os três participantes do Grupo 2 responderam prontamente após serem expostos ao procedimento de treino por encadeamento e com atraso no de pares de estímulos adjacentes. No Grupo 1, um participante (RLS) respondeu com atraso quando exposto ao treino com pares de estímulos adjacentes. Após exposição ao treino por encadeamento, o mesmo foi o único que manteve um desempenho estável, mesmo com atraso. e 5), após o treino de sobreposição com pares de 


\begin{tabular}{|c|c|c|c|c|}
\hline Tipo de tentativa de Treino & Tipo de tentativa de Testes & RUG & HSN & RLS \\
\hline $\mathrm{X} 1 \rightarrow \mathrm{X} 2$ & & $6 / 6$ & $6 / 6$ & $6 / 6$ \\
\hline \multirow{23}{*}{$\begin{aligned} & \mathrm{X} 1 \rightarrow \mathrm{X} 2 \rightarrow \mathrm{X} 3 \\
& \mathrm{X} 1 \stackrel{\mathrm{X} 2}{\rightarrow} \rightarrow \mathrm{X} 3 \rightarrow \mathrm{X} 4 \\
& \mathrm{X} 1 \rightarrow \mathrm{X} 2 \rightarrow \mathrm{X} 3 \rightarrow \mathrm{X} 4 \rightarrow \mathrm{X} 5\end{aligned}$} & & $9 / 9$ & $9 / 9$ & $9 / 9$ \\
\hline & & $12 / 12$ & $12 / 12$ & $12 / 12$ \\
\hline & (seqüenciacão) & $15 / 15$ & $15 / 15$ & $15 / 15$ \\
\hline & 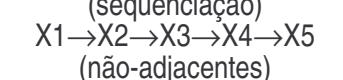 & $1 / 2$ & $1 / 2$ & $1 / 2$ \\
\hline & $\mathrm{X} 1 \rightarrow \mathrm{X3}$ & $1 / 2$ & $1 / 2$ & $1 / 2$ \\
\hline & $\mathrm{X} 1 \rightarrow \mathrm{X} 4$ & $1 / 2$ & $1 / 2$ & $1 / 2$ \\
\hline & $\mathrm{X} 1 \rightarrow \mathrm{X} 5$ & $1 / 2$ & $1 / 2$ & $1 / 2$ \\
\hline & $\mathrm{X} 2 \rightarrow \mathrm{X} 4$ & $1 / 2$ & $1 / 2$ & $1 / 2$ \\
\hline & $\mathrm{X} 2 \rightarrow \mathrm{X} 5$ & $1 / 2$ & $1 / 2$ & $1 / 2$ \\
\hline & $\mathrm{X} 3 \rightarrow \mathrm{X} 5$ & $1 / 2$ & $1 / 2$ & $1 / 2$ \\
\hline & & $6 / 6$ & $6 / 6$ & $6 / 6$ \\
\hline & & $9 / 9$ & $9 / 9$ & $9 / 9$ \\
\hline & & $12 / 12$ & $12 / 12$ & $12 / 12$ \\
\hline & & $15 / 15$ & $15 / 15$ & $15 / 15$ \\
\hline & $\begin{array}{c}\text { (seqüenciação) } \\
\mathrm{W} 1 \rightarrow \mathrm{W} \rightarrow \mathrm{W} \rightarrow \mathrm{W} \rightarrow \mathrm{W} 4 \rightarrow \mathrm{W} 5 \\
\text { (não-adjacentes) }\end{array}$ & $1 / 2$ & $1 / 2$ & $1 / 2$ \\
\hline & $\mathrm{W} 1 \rightarrow \mathrm{W} 3$ & $1 / 2$ & $1 / 2$ & $1 / 2$ \\
\hline & $\mathrm{W} 1 \rightarrow \mathrm{W} 4$ & $1 / 2$ & $1 / 2$ & $1 / 2$ \\
\hline & $\mathrm{W} 1 \rightarrow \mathrm{W} 5$ & $1 / 2$ & $1 / 2$ & $1 / 2$ \\
\hline & $\mathrm{W} 2 \rightarrow \mathrm{W} 4$ & $1 / 2$ & $1 / 2$ & $1 / 2$ \\
\hline & $\mathrm{W} 2 \rightarrow \mathrm{W} 5$ & $1 / 2$ & $1 / 2$ & $1 / 2$ \\
\hline & $\begin{array}{c}\text { W3 } \rightarrow \text { W5 } \\
\text { (substitutabilidade) }\end{array}$ & $1 / 2$ & $1 / 2$ & $1 / 2$ \\
\hline & $\mathrm{W} 1 \rightarrow \mathrm{X} 2 \rightarrow \mathrm{W} 3 \rightarrow \mathrm{X} 4 \rightarrow \mathrm{W} 5$ & $0 / 2$ & 2/2 & $2 / 2$ \\
\hline & $\mathrm{X} 1 \rightarrow \mathrm{W} 2 \rightarrow \mathrm{X} 3 \rightarrow \mathrm{W} 4 \rightarrow \mathrm{X} 5$ & $0 / 2$ & $2 / 2$ & $2 / 2$ \\
\hline
\end{tabular}

Tabela 5 - Número de respostas corretas pelo número máximo de tentativas durante o treino e testes com pares de estímulos adjacentes e não adjacentes e testes de substitutabilidade

GRUPO 2

ENCADEAMENTO

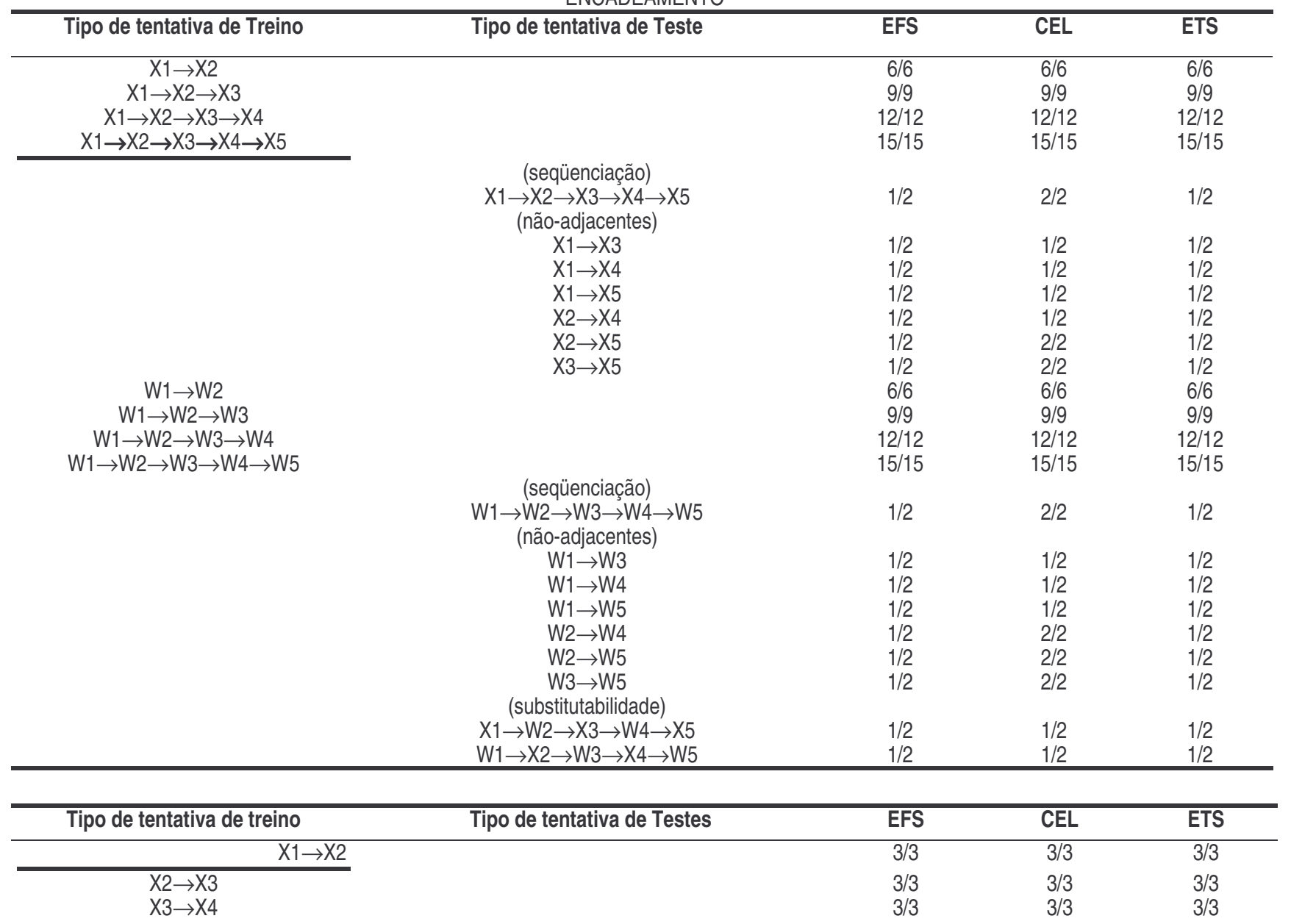




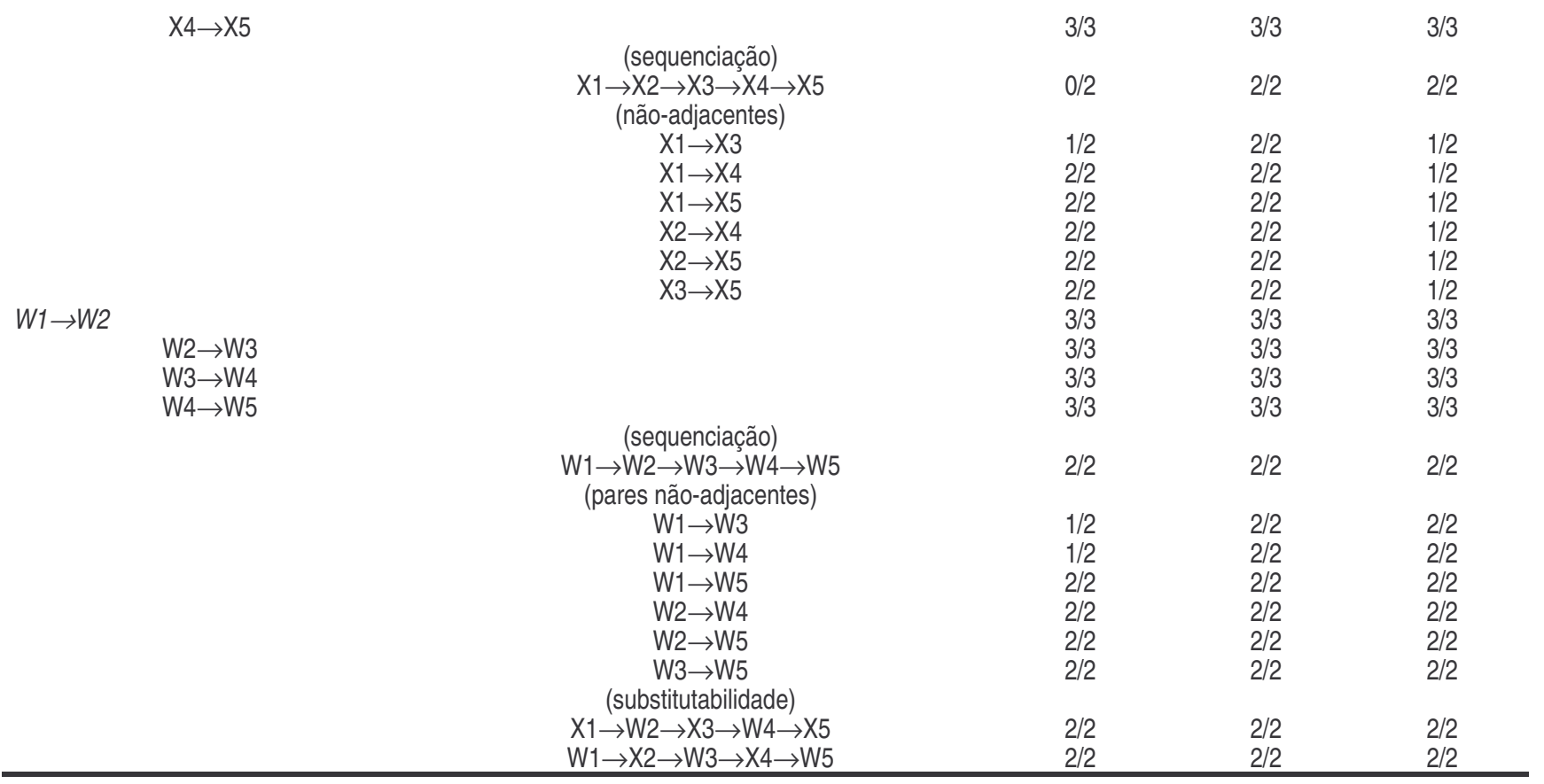

Esses resultados parecem demonstrar que o treino por encadeamento foi mais eficiente do que o treino de sobreposição com pares de estímulos adjacentes, com os participantes respondendo prontamente às condições experimentais de testes, especialmente no de substitutabilidade. Pode-se observar ainda um efeito positivo na ordem de treino por encadeamento nos testes de substitutabilidade no Grupo 2, com todos os participantes respondendo positivamente mesmo com atraso.

A contribuição do presente estudo está em demonstrar que a introdução gradual dos estímulos durante o treino por encadeamento, ampliando-se cada sequiência gradativamente, facilitou a emergência de classes seqüenciais mais prontamente. A presença simultânea dos cinco estímulos na seqüência mais longa, diferentemente das contingências de reforçamento usadas durante o treino de sobreposição com pares de estímulos adjacentes parece ter facilitado a permutabilidade dos estímulos.

Entretanto, o estudo não permitiu verificar se as classes de estímulos estabelecidas a partir da mediação de uma resposta comum, ou seja, a seleção dos estímulos em uma determinada ordem, satisfaz o critério de equivalência apresentado por Sidman e Tailby (1982). Porém, os estudos (Sidman, 1994) apontam que substitutabilidade de estímulos caracteriza a formação de classes de estímulos equivalentes. Os resultados do presente estudo permitem inferir a propriedade de transitividade, a partir dos testes com pares de estímulos nãoadjacentes. Além disso, os resultados mais relevantes foram a emergência imediata de classes seqüenciais apresentadas pelos três alunos do Grupo 2 e posteriormente a emergência com atraso após treino de sobreposição com pares de estímulos adjacentes. Portanto, esses resultados estão baseados nos pressupostos sugeridos por Green e cols. (1993) para a estruturação de testes averiguam as propriedades de relações ordinais, estabelecidas entre estímulos em sequiências e entre seqüências independentes.

Uma outra variável não menos relevante identificada nesse estudo refere-se à natureza dos estímulos. Os estímulos utilizados aqui (aves e mamíferos) de alguma forma já faziam parte do repertório dos alunos e isso também pode ter contribuído para os resultados, por conta do efeito de histórias diferentes de reforçamento que cada aluno traz para o ambiente experimental. Um estudo que investigasse a natureza dos estímulos (por exemplo, com formas abstratas) com os dois procedimentos de ensino aqui utilizados, poderia replicar sistematicamente esses resultados ou identificar novas fontes de controle do comportamento por relações de ordem. Uma outra possibilidade seria a análise de comportamentos conceitualmente numéricos. $\mathrm{Na}$ formação de classes ordinais e não simplesmente seqüenciais (ver Carmo, 2002), uma resposta poderia estar sob controle de propriedades dos estímulos, por exemplo, "maior que" ou "menor que", onde o pesquisador estaria ensinando comportamentos mais socialmente relevantes, diferentemente daquela estabelecida nos estudos descritos pela literatura (ver Mackay, Stromer \& Serna, 1998), cuja base de controle do responder estava simplesmente na posição ocupada pelo estímulo na sequiência.

\section{REFERÊNCIAS}

American Psychiatric Association (2000). Diagnostic and Statistical Manual of Mental Disorders (DSM-IV-revised), $4^{\mathrm{a}}$ edition. Washington, DC: Author.

Carmo, J. S. (2002). Definições operacionais de habilidades matemáticas elementares. Em H. J. Guilhardi; M. B. B. P. Madi; P. P. Queiroz \& M. C. Scoz (Orgs.), Sobre comportamento e cognição: contribuições para a construção da teoria do comportamento. Santo André: ESETec editores associados.

Dunn, L. M. \& Dunn, I. M. (1981). Peabody Picture Vocabulary Test-Revised. Circle Pines, MN: American Guidance Service. 
Green, G.; Stromer, R. \& Mackay, H. (1993). Relational learning in stimulus sequences. The Psychological Record, 43, 599616.

Holcomb, W. L.; Stromer, R. \& Mackay, H. A. (1997). Transitivity and emergent sequence performance in young children. Journal of Experimental Child Psychology, 65, 96124.

Lazar, R. (1977). Extending sequence-class membership with matching to sample. Journal of the Experimental Analysis of Behavior, 27, 381-392.

Lima, M. P. \& Assis, G. J. A. (2003). Emergência de classes seqüenciais após treino com pareamento consistente. Psicologia: Teoria e Pesquisa, 19 (1), 75-84.

Mackay, H. A.; Stromer, R. \& Serna, R. W. (1998). Emergent behavior and intellectual functioning: stimulus classes, generalization, and transfer. Em: S. Soraci \& W. J. Mallvane (Orgs.), Perspectives on fundamental Processes in intellectual functioning (pp. 287-310). Norwood, NJ: Ablex.

Portella, I. V. M.; Sampaio, M. E. C.; Baptista, M. Q. G \& Assis, G. J. A. (2003). Em busca de relacões ordinais sob controle condicional em portadores de deficiência mental. Resumos de Comunicação Científica do XII Encontro Brasileiro de Psicoterapia e Medicina Comportamental (ABPMC). Londrina-PR

Sampaio, M. E. C. \& Assis, G. J. A. (no prelo). Equivalência de estímulos após formação de classes seqüenciais em pessoas portadoras de necessidades educacionais. Acta Comportamentalia.

Sidman, M. (1960). Tactics of Scientific Research. New York: Basic Books.

Sidman, M. (1986). Functional Analysis of Emergent Verbal Classes. Em T. Thompson \& M. D. L. Zeiler (Orgs.), Analysis and Integration of Behavioral Units (pp. 213-245). N. J.: Lawrence Erlbaum Associates Publishers.

Sidman, M. (1994). Equivalence Relations and Behavior: A Research Story. Boston: Authors Cooperative, Inc. Publishers.

Sidman, M. \& Tailby, W. (1982). Conditional discrimination vs. matching to sample: An expansion of the testing paradigm. Journal of the Experimental Analysis of Behavior, 37, 5-22.

Sigurdardottir, Z. G.; Green, G. \& Saunders, R. R. (1990). Equivalence classes generated by sequence training. Journal of the Experimental Analysis of Behavior, 53, 47-63.

Stromer, R. \& Mackay, H. A. (1990). A note on the study of transitive relation in stimulus sequence. Experimental Analysis of Human Behavior Bulletin, 8, 2-5.

Stromer, R. \& Mackay, H. A. (1992). Conditional stimulus control of children's sequence production. Psychological Reports, 70, 903-912.

Stromer, R. \& Mackay, H. A. (1993). Human sequential behavior: Relations among stimuli, class formation, and derived sequences. The Psychological Record, 43, 107-131.

Stromer, R.; Mackay, H. A.; Cohen, M. \& Stoddard, L. T. (1993). Sequence learning in individuals with behavioral limitations. Journal of Intellectual Disability Research, 37, 243-261. 


\section{Nota:}

${ }^{1}$ O presente trabalho contou com financiamento da Universidade Federal do Pará (PROINT).

\section{Sobre os autores:}

Grauben José Alves de Assis: Doutor em Psicologia Experimental pela USP; docente do Departamento de Psicologia Experimental da UFPA; membro do Programa de Pós-Graduação em Teoria e Pesquisa do Comportamento -Universidade Federal do Pará; bolsista 2 b do CNPq.

Maria Elizângela Carvalho Sampaio: Mestre e Doutoranda pelo Programa de Pós-Graduação em Teoria e Pesquisa do Comportamento/UFPA.

Endereço para correspondência: TV. Castelo Branco, 1923/301 - Bairro do Guamá, Belém-Pará. - CEP $66063-420$ Fone/FAX: (91) 211-1662 - E-mail: gjaa@cpgp.ufpa.br 